\title{
Determinants of the intercept and slope of glomerular filtration rate in recipients of a live donor kidney transplant
}

\author{
Martina Hamböck - Anton Staudenherz - Alexander Kainz - Barbara Geist - Manfred Hecking • \\ Konstantin Doberer · Marcus Hacker · Georg A. Böhmig (D)
}

Received: 25 July 2019 / Accepted: 10 January 2020 / Published online: 3 February 2020

(C) The Author(s) 2020

\begin{abstract}
Summary
Background Donor kidney function is considered a critical determinant of allograft survival after live donor (LD) kidney transplantation, but its independent impact on the evolution of graft function is less well defined. The objective of this study was to dissect the relative contribution of LD kidney function to baseline estimated glomerular filtration rate (eGFR) of recipients and its decline.

Methods In this study 91 LD kidney transplantations performed between 2007 and 2015 were included. The eGFR of donated kidneys (eGFR-dk) was calculated from total LD eGFR (eGFR-dt) based on the results of isotope nephrography. Recipient eGFR (eGFR-r) determined 6-monthly until 36 months posttransplantation served as dependent variable in mixed linear models estimating changes in baseline allograft function (intercept) and eGFR-r slope. Models were adjusted either for eGFR-dk or eGFR-dt, in addition to other potential confounders.

Results Overall, unadjusted mean eGFR-r at baseline (6 months) and its annual decline in allograft func-
\end{abstract}

\footnotetext{
M. Hamböck, MD, PhD · A. Staudenherz, MD · B. Geist, PhD M. Hacker, MD

Division of Nuclear Medicine, Department of Biomedical Imaging and Image-guided Therapy, Medical University of Vienna, Vienna, Austria

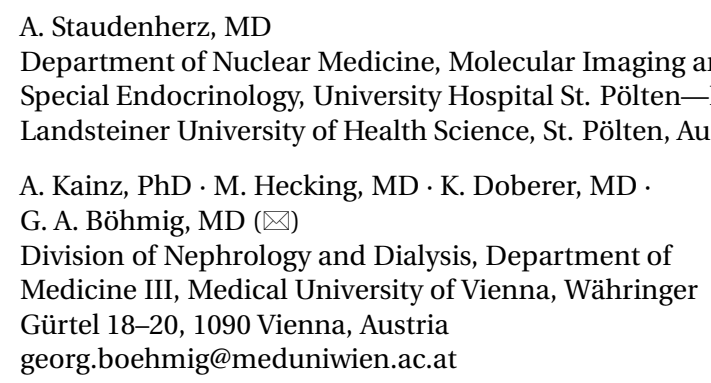

georg.boehmig@meduniwien.ac.at

tion were $56.5 \mathrm{~mL} / \mathrm{min} / 1.73 \mathrm{~m}^{2}$ and $-0.2 \mathrm{~mL} / \mathrm{min} / 1.73$ $\mathrm{m}^{2}$, respectively. In multivariate analysis, eGFR$\mathrm{dk}$ impacted on baseline eGFR-r $(0.6 \mathrm{~mL} / \mathrm{min} / 1.73$ $\mathrm{m}^{2}$ mean estimated increase per unit; $P=0.02$ ) but not on its slope. In the eGFR-dt-adjusted model, a marginal effect was observed for LD age $(P=0.05)$. Both models identified antibody-mediated rejection (ABMR) as the strongest risk factor of accelerated loss of allograft function (eGFR-r slope: approximately $-6 \mathrm{~mL} / \mathrm{min} / 1.73 \mathrm{~m}^{2}$ per year; $P \leq 0.02$ ).

Conclusion Donor-related characteristics, most prominently the function of donated kidneys and LD age, were predictive of eGFR at baseline. The ABMR was identified as the cardinal cause of progressive deterioration of allograft function.

Keywords Antibody-mediated rejection - Donor age Estimated glomerular filtration rate $\cdot$ Isotope nephrography $\cdot$ Kidney transplantation

\section{Introduction}

Live donor (LD) kidney transplantation is considered the best treatment option for patients with end-stage renal disease (ESRD), allowing superior outcomes in terms of patient survival, quality of life and healthrelated expenses. Careful LD selection, however, is essential to ensure best possible treatment results and maximum safety for both donors and recipients.

To facilitate the work-up of potential kidney donors, a variety of national and international guidelines have been formulated, most of them agreeing that glomerular filtration rate (GFR) should be evaluated with direct measurements of exogenous filtration markers, in addition to serum creatinine-based estimations of GFR [1, 2]. Levels of kidney function accepted for donation need to be adapted to the individual risk profile, but for individuals with a GFR of 
Fig. 1 Study flow chart. Abbreviations: eGFR estimated glomerular filtration rate, $L D$ living donor, 99mTc-MAG3 Tc-99m-mercaptoacetyltriglycine acid

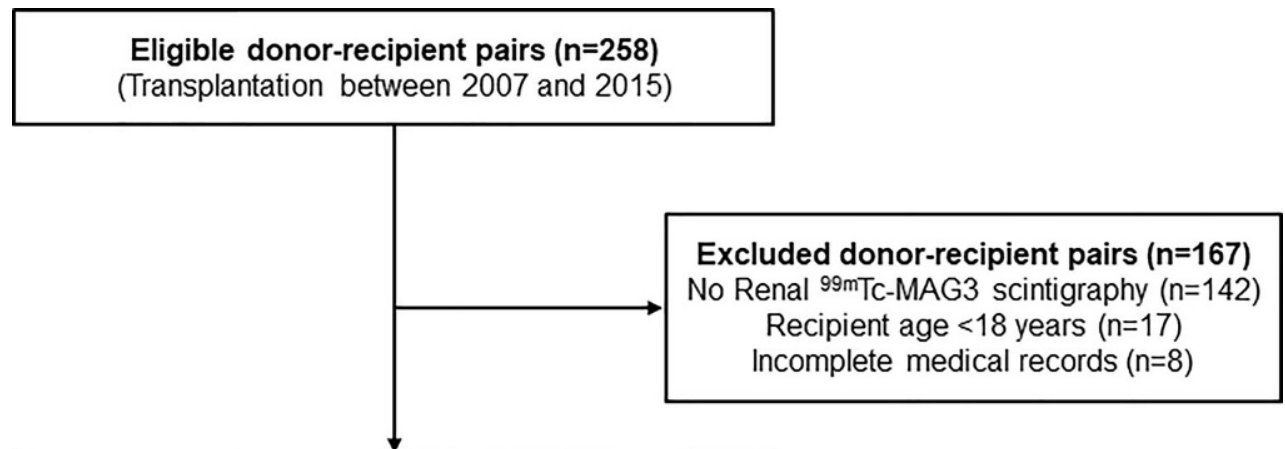

Study population ( $n=91$ )

Adult living kidney donors and recipients

Valid scintigraphic data and complete medical records<smiles>C1CCCCC1</smiles>

Evaluation of

total LD eGFR and the eGFR of the donated kidney

in relation to:

(i) Recipient baseline eGFR (at 3 months)

(ii) Recipient eGFR slope (over 12 months)
$>90 \mathrm{~mL} / \mathrm{min}$ per $1.73 \mathrm{~m}^{2}$ it is generally considered safe to donate. In addition, isotope nephrography (ING) may help to determine the relative function of the kidneys supporting the choice of the nephrectomy side $[1,2]$.

In recent years, there has been a trend towards the acceptance of significant LD comorbidities, especially in older individuals, provided that the lifetime risk for the development of chronic kidney disease is low [3]. This may also include donors with a GFR below the commonly accepted thresholds [4]. The use of marginal donor kidneys, however, may impact substantially on allograft performance. A prominent risk factor in this respect was shown to be LD age. Large cohort studies have revealed inferior short-term and long-term outcomes for organs originating from older donors [5-10]. Results are consistent with observations made in deceased donor (DD) kidney transplantation, where organ allocation is supported by agematching algorithms, which take the metabolic demand of recipients into account [11].

The relative outcome effect of predonation kidney function in LD transplantation is less well studied. Norden et al. [12] observed an increased risk for graft loss in a population of 344 LD kidney transplant recipients, when donors had an unadjusted GFR below $80 \mathrm{~mL} / \mathrm{min}$. This finding is supported by a systematic review of seven studies, demonstrating associations of higher donor GFR with superior allograft function and transplant survival [13]. Definitions of GFR, however, were heterogeneous, there was no adjustment for relevant confounders, and a possible influence of unequal functional distribution between the donated and re- maining kidneys was not taken into account. Moreover, none of these studies included detailed analyses of the slope of recipient estimated glomerular filtration rate (eGFR), which, confounded by a variety of immunological and non-immunological factors, may serve as a useful surrogate endpoint predicting longterm renal allograft survival $[14,15]$.

In this retrospective cohort study the independent impact of LD kidney function on recipient eGFR at baseline (intercept) and its slope calculated from serial eGFR measurements was investigated during the first 3 post-transplantation years. To add accuracy to the analysis the eGFR of the donated kidneys was separately calculated based on the results of Tc-99mmercaptoacetyltriglycine acid ( $\left.{ }^{99 m} \mathrm{Tc}-\mathrm{MAG} 3\right)$ scintigraphy. Mixed linear models were applied to quantify the impact of LD kidney function on allograft performance, in the context of other potentially outcomerelated variables.

\section{Materials and methods}

\section{Study design and patients}

The primary aim of this retrospective single center cohort study was to dissect the contribution of LD kidney function, as reflected by the (i) eGFR of the donated kidney (eGFR-dk) or (ii) total donor eGFR (eGFR-dt), to baseline allograft function at 6 months (intercept) and its course until 36 months post-transplantation (slope). The study included 91 out of 258 LD allograft recipients at the Vienna transplantation unit between January 2007 and December 2015. In- 
clusion criteria were a recipient age of $\geq 18$ years, the availability of ING-based split function of donated kidneys and a complete follow-up until April 2018, including serial recipient eGFR (eGFR-r) measurements at hospital discharge and at 6, 12, 18, 24 and 36 months after transplantation. Of the recipients 167 did not meet these criteria and were excluded from the analysis. A flow chart of the study is provided in Fig. 1. The study was approved by the institutional ethics committee (No. 2252/2017) and was carried out in accordance with the principles of the Declaration of Helsinki 2008 and the Declaration of Istanbul.

\section{Evaluation of kidney function}

Estimated GFR was calculated using the Chronic Kidney Disease Epidemiology Collaboration (CKD-EPI) equation [16]. Until February 2012, 24-h urine creatinine clearance was evaluated for LD selection. Thereafter, the donor work-up included the assessment of measured GFR (mGFR) using chromium-51 ethylenediaminetetraacetic acid ( ${ }^{51} \mathrm{Cr}$-EDTA). Donors received approximately $2 \mathrm{MBq}$ of the radiolabelled filtration marker, and sequentially drawn blood samples (120, 180 and $240 \mathrm{~min}$ after administration) served to determine the plasma clearance. Body surface area-adjusted GFR values were calculated with in-house software, as described by Geist et al. [17]. According to our local standard, a creatinine clearance or an adjusted mGFR below $80 \mathrm{~mL} / \mathrm{min}$ was considered to be a contraindication for donor nephrectomy.

\section{Isotope nephrography}

Renal ${ }^{99 m}$ Tc-MAG3 scintigraphy to determine the relative functional distribution between the two donor kidneys (split kidney function) was performed according to the protocol of the European Association of Nuclear Medicine [18]. Image acquisition was performed with a gamma camera, as previously described [19]. The imaging software HERMES Gold ${ }^{\mathrm{TM}}$ (Hermes Medical Solutions AB, Stockholm, Sweden) was used to draw regions of interest (ROIs) around the kidneys, the heart and the perirenal background. The mean transit time (MTT) and the relative kidney function from $1 \mathrm{~min}$ to $3 \mathrm{~min}$ were extracted from the integrals of renal time activity curves (TACs). The LD candidates with a side difference of $>20 \%$ ( $>60 \%$ vs. $<40 \%$ ) were not accepted for donation. The relative function determined by renal MAG3 scintigraphy was used to calculate eGFR-dk and the mGFR of the donated kidney (mGFR-dk) by its multiplication with eGFR-dt or total LD mGFR (mGFR-dt), respectively. The eGFR of the remaining kidney (eGFR-rk) was calculated by subtraction of eGFR-dk from eGFR-dt. MTT values of 1.9-2.9 min were considered normal [20].

\section{Immunosuppression}

The majority of included recipients (89\%) received calcineurin inhibitor-based maintenance immunosuppression, commonly triple therapy including tacrolimus, mycophenolic acid and steroids (Table 1). Most recipients (90\%) also received interleukin (IL)2 receptor antibody induction. During follow-up maintenance immunosuppression was changed in 14 of the patients (tacrolimus to cyclosporin A: $n=4$; tacrolimus to sirolimus or everolimus: $n=4$; belatacept to tacrolimus: $n=3$; sirolimus or everolimus to tacrolimus: $n=2$; cyclosporin A to tacrolimus: $n=1$ ). Median tacrolimus trough levels were $7.7 \mathrm{ng} / \mathrm{mL}$ and $6.3 \mathrm{ng} / \mathrm{mL}$ after 6 and 12 months, respectively. Of the patients eight were transplanted across major $\mathrm{ABO}$ barriers, following a course of $\mathrm{ABO}$ antigenspecific $(n=6)$ or semi-selective (in cases of additional preformed anti-HLA donor-specific antibodies, DSA: $n=2$ ) immunoadsorption and a single dose of rituximab and intravenous immunoglobulin (IVIG).

\section{Transplant biopsies}

Indication biopsies were performed for graft dysfunction and/or significant proteinuria. Our standard did not include surveillance biopsies. Histomorphology and immunohistochemistry were evaluated on formalin-fixed paraffin-embedded sections. T cell-mediated rejection (TCMR) and antibody-mediated rejection (ABMR) were defined according to the 2015 update of the Banff classification of renal allograft pathology [21].

\section{Statistical analysis}

Continuous data were expressed as median and interquartile range (IQR) and categorical variables as absolute and relative frequencies. Kaplan-Meier analysis was applied for calculation of graft and patient survival. The influence of LD kidney function on baseline eGFR-r and on its slope was evaluated using mixed linear models. We calculated two separate models, in which LD kidney function was either characterized by eGFR-dk or by eGFR-dt and LD kidney function and time were included in each calculation. Slope estimates additionally considered interactions of variables with time. In the reduced model, several other donor and recipient variables were added one by one. The multivariable model was expanded by variables with a $P$ value of $<0.157$ for their impact on baseline eGFR-r or its slope in the reduced model [22]. Levels of eGFR-r from 6 months to 36 months were used as dependent variables. For correlation analysis, Spearman's rank correlation test was applied. A 2sided $P<0.05$ was considered significant. For statistical analysis, IBM SPSS Statistics 23 for Mac (IBM Corporation, Armonk, NY, USA) and SAS 9.4 for Windows (The SAS Institute Inc., Cary, NC, USA) were used. 
Table 1 Recipient baseline characteristics

\begin{tabular}{|c|c|}
\hline Parameters & Total $(n=91)$ \\
\hline \multicolumn{2}{|l|}{ Variables recorded at the time of transplantation } \\
\hline Age, years, median (IQR) & $42.4(28.1-54.1)$ \\
\hline Female sex, $n(\%)$ & $32(35.2)$ \\
\hline \multicolumn{2}{|l|}{ Underlying kidney disease, $n(\%)$} \\
\hline Glomerulonephritis & $39(42.9)$ \\
\hline Polycystic kidney disease & $18(19.8)$ \\
\hline Obstructive nephropathy & $11(12.1)$ \\
\hline Diabetic nephropathy & $3(3.3)$ \\
\hline Hypertensive nephropathy & $1(1.1)$ \\
\hline Other, unknown & $19(20.9)$ \\
\hline Preemptive transplantation, $n(\%)$ & $28(30.8)$ \\
\hline Recipient of a retransplant, $n(\%)$ & $10(11)$ \\
\hline ABO/HLA-incompatible transplantation, $n(\%)^{\mathrm{a}}$ & $10(11)$ \\
\hline Sum of HLA mismatch in A, B and DR, median (IQR) & $3(2-4)$ \\
\hline \multicolumn{2}{|l|}{ Baseline immunosuppression, $n(\%)$} \\
\hline Induction with IL-2R antibody & $82(90.1)$ \\
\hline Tacrolimus & $75(82.4)$ \\
\hline Trough level at 6 months, ng/ml, median (IQR) & $7.7(6.0-9.1)$ \\
\hline Trough level at 12 months, ng/ml, median (IQR) & $6.3(5.2-8.0)$ \\
\hline Cyclosporin A & $6(6.6)$ \\
\hline mTOR inhibitor ${ }^{b}$ & $3(3.3)$ \\
\hline Belatacept & $6(6.6)$ \\
\hline Mycophenolic acid & $88(96.7)$ \\
\hline Azathioprine & $1(1.1)$ \\
\hline Steroid & $90(98.9)$ \\
\hline \multicolumn{2}{|c|}{$\begin{array}{l}\text { IL-2R interleukin-2 receptor, IQR interquartile range, } m T O R \text { mammalian } \\
\text { target of rapamycin } \\
\text { aEight patients were transplanted across ABO and two patients across both } \\
\text { ABO and HLA-donor-specific antibody (DSA) barriers } \\
\text { bSirolimus or everolimus }\end{array}$} \\
\hline
\end{tabular}

\section{Results}

\section{Patient characteristics}

The study included 91 adult recipients of a LD kidney allograft. Key inclusion criteria were a detailed INGbased LD work-up and a complete recipient followup.

Baseline donor and recipient data are provided in Tables 1 and 2, respectively. The median recipient age was 42 years and $35 \%$ of the patients were female. The most common causes of ESRD were glomerulonephritis and polycystic kidney disease, $31 \%$ of the patients underwent preemptive transplantation and $11 \%$ were recipients of a re-transplant. The median sum of HLA mismatches in A, B and DR was three (Table 1).

The LD were in median 52 years old, and $63 \%$ were female and $53 \%$ of the donors were living-related. Evaluation of LD kidney function revealed a median eGFR-dt of $87 \mathrm{~mL} / \mathrm{min} / 1.73 \mathrm{~m}^{2}$ and a median $\mathrm{mGFR}$-dt of $120 \mathrm{~mL} / \mathrm{min} / 1.73 \mathrm{~m}^{2}$, respectively. The ING-based analysis revealed a median MTT of $2.9 \mathrm{~min}$ and a median relative organ function of $51 \%$
Table 2 Results of LD kidney evaluation

\begin{tabular}{|c|c|}
\hline Parameters & Total $(n=91)$ \\
\hline \multicolumn{2}{|l|}{ Variables recorded at the time of donation } \\
\hline Age, years, median (IQR) & $51.6(44.2-57.2)$ \\
\hline Female sex, $n(\%)$ & $57(62.6)$ \\
\hline $\mathrm{BMI}, \mathrm{kg} / \mathrm{m}^{2}$, median (IQR) & $25.6(22.9-28.7)$ \\
\hline Living-related, $n$ (\%) & $53(58.2)$ \\
\hline Donation of left kidney, $n(\%)$ & $73(80.2)$ \\
\hline $\begin{array}{l}\text { Total eGFR (eGFR-dt), } \mathrm{mL} / \mathrm{min} / 1.73 \mathrm{~m}^{2} \text {, median } \\
\text { (IQR) }\end{array}$ & $87(77-98)$ \\
\hline $\begin{array}{l}\text { Total mGFR (mGFR-dt), mL/min/1.73 m², median } \\
(\text { IQR) }\end{array}$ & $120(104-139)$ \\
\hline \multicolumn{2}{|l|}{ ING-based parameters of donated kidney, median (IQR) } \\
\hline Mean transit time (MTT), min & $2.9(2.6-3.3)$ \\
\hline Relative function, \% & $51(48-54)$ \\
\hline $\begin{array}{l}\text { eGFR according to relative function (eGFR-dk), } \\
\mathrm{mL} / \mathrm{min} / 1.73 \mathrm{~m}^{2}\end{array}$ & $43(38-50)$ \\
\hline $\begin{array}{l}\mathrm{mGFR} \text { according to relative function (mGFR-dk) } \\
\mathrm{mL} / \mathrm{min} / 1.73 \mathrm{~m}^{2} \text {, }\end{array}$ & $62(51-71)$ \\
\hline \multicolumn{2}{|c|}{$\begin{array}{l}\text { BMI body mass index, eGFR estimated glomerular filtration rate, ING isotope } \\
\text { nephrography, IQR interquartile range, } L D \text { live donor, mGFR measured } \\
\text { glomerular filtration rate } \\
\text { amGFR measurements were available for } 53 \text { donors } \\
\text { beGFR-dk and mGFR-dk were calculated from eGFR-dt or mGFR-dt on the } \\
\text { basis of the relative kidney function determined in ING }\end{array}$} \\
\hline
\end{tabular}

for the donated kidneys, of which $80.2 \%$ were left kidneys. Median eGFR-dk and mGFR-dk were 43 and $62 \mathrm{~mL} / \mathrm{min} / 1.73 \mathrm{~m}^{2}$, respectively (Table 2).

\section{Allograft and recipient outcomes}

Transplant outcomes are detailed in Table 3. The course of eGFR-r until 36 months posttransplantation is illustrated in Fig. 2. In the overall cohort, the unadjusted mean baseline eGFR-r at 6 months (intercept) was $56.5 \mathrm{~mL} / \mathrm{min} / 1.73 \mathrm{~m}^{2}$ (95\% CI: $52.3-60.7 \mathrm{~mL} / \mathrm{min} /$ $1.73 \mathrm{~m}^{2}$ ), and the unadjusted mean annual decline in allograft function (slope) was $-0.2(-1.8-1.3)$ $\mathrm{mL} / \mathrm{min} / 1.73 \mathrm{~m}^{2}$.

Levels of eGFR-r at hospital discharge correlated with predonation LD eGFR (Fig. 3). Correlations were stronger if donor kidney function was characterized by the eGFR-dk than the eGFR-dt (rho $=0.32$ versus rho $=0.23$ ). Moreover, there was a close correlation between predonation LD eGFR, expressed as eGFRdt or eGFR of the remaining kidney (eGFR-rk), and postdonation LD eGFR (rho=0.65) (Fig. 3).

The most common histopathological findings in indication biopsies were TCMR $(n=18)$ and ABMR $(n=10)$. Following the Banff 2015 scheme, 3 recipients were diagnosed with acute active ABMR, and 7 recipients with chronic active ABMR (Table 3 ).

The 1-year, 3-year and 5-year death-censored graft survival rates were $100 \%, 98 \%$ and $95 \%$, respectively (Fig. 2). Of the patients 9 lost their transplants after a median interval of 5.7 years, most commonly (6 cases) as a result of ABMR (BK virus nephropathy: $n=1$; unknown cause: $n=2$ ). Patient survival at 1 , 
Table 3 Transplant outcomes

\begin{tabular}{|c|c|}
\hline Parameters & Total $(n=91)$ \\
\hline \multicolumn{2}{|l|}{ Variables recorded after transplantation } \\
\hline \multicolumn{2}{|c|}{ Recipient eGFR (eGFR-r), mL/min/1.73 m², median (IQR) ${ }^{\mathrm{a}}$} \\
\hline At discharge ${ }^{b}$ & $57(46-72)$ \\
\hline 6 months & $56(44-67)$ \\
\hline 12 months & $60(47-70)$ \\
\hline 24 months & $55(45-71)$ \\
\hline 36 months & $55(44-67)$ \\
\hline \multicolumn{2}{|c|}{ Histopathological findings in indication biopsies, $n(\%)$} \\
\hline TCMR & $18(19.8)$ \\
\hline ABMR & $10(11)$ \\
\hline De novo/recurrent glomerulonephritis & $4(4.4)$ \\
\hline BK virus nephropathy & $3(3.3)$ \\
\hline \multicolumn{2}{|l|}{ Death-censored graft survival, $\%^{c}$} \\
\hline 1 year & 100 \\
\hline 3 years & 98 \\
\hline 5 years & 95 \\
\hline \multicolumn{2}{|l|}{ Patient survival, \% } \\
\hline 1 year & 100 \\
\hline 3 years & 98 \\
\hline 5 years & 98 \\
\hline \multicolumn{2}{|c|}{ 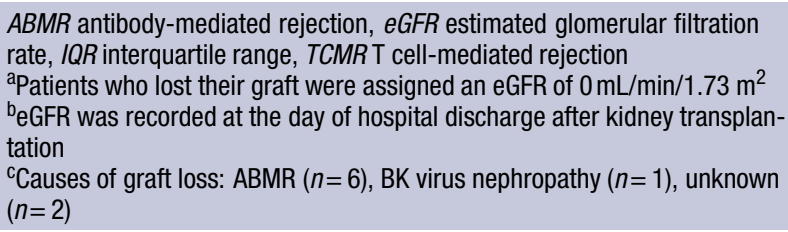 } \\
\hline
\end{tabular}

3 and 5 years was $100 \%, 98 \%$ and $98 \%$, respectively (Table 3). Overall, three deaths were recorded during follow-up (two with a functioning allograft).

\section{Effect of donor kidney function on recipient eGFR}

We applied two separate mixed linear models to characterize the effects of LD kidney function on eGFR-r. The first model (Table 4) was adjusted for eGFR-dk and other relevant donor- or recipient-related variables. Multivariable analysis revealed a significant impact of eGFR-dk on eGFR-r at baseline (0.6mL/min $/ 1.73 \mathrm{~m}^{2}, 95 \%$ CI: $0.1-1.1 \mathrm{~mL} / \mathrm{min} / 1.73 \mathrm{~m}^{2}$ mean estimated increase per unit; $P=0.02$ ) but not on eGFR-r slope $(P=0.27)$. The ABMR was the strongest predictor of eGFR-r slope (mean estimated annual decline: $-5.8(-10.4$ to -1.2$\left.) \mathrm{mL} / \mathrm{min} / 1.73 \mathrm{~m}^{2} ; P=0.01\right)$. We also observed a marginal effect of donor body mass index (BMI; $P=0.04)$. Other variables selected for multivariate analysis, including LD age, donor and recipient gender, baseline immunosuppression or MTT, however, had no significant effect. Notably, also pre-emptive transplantation was not associated with allograft function.

The second model (Table 5) included the same variables but was adjusted for eGFR-dt. There was no significant effect of eGFR-dt on eGFR-r at baseline
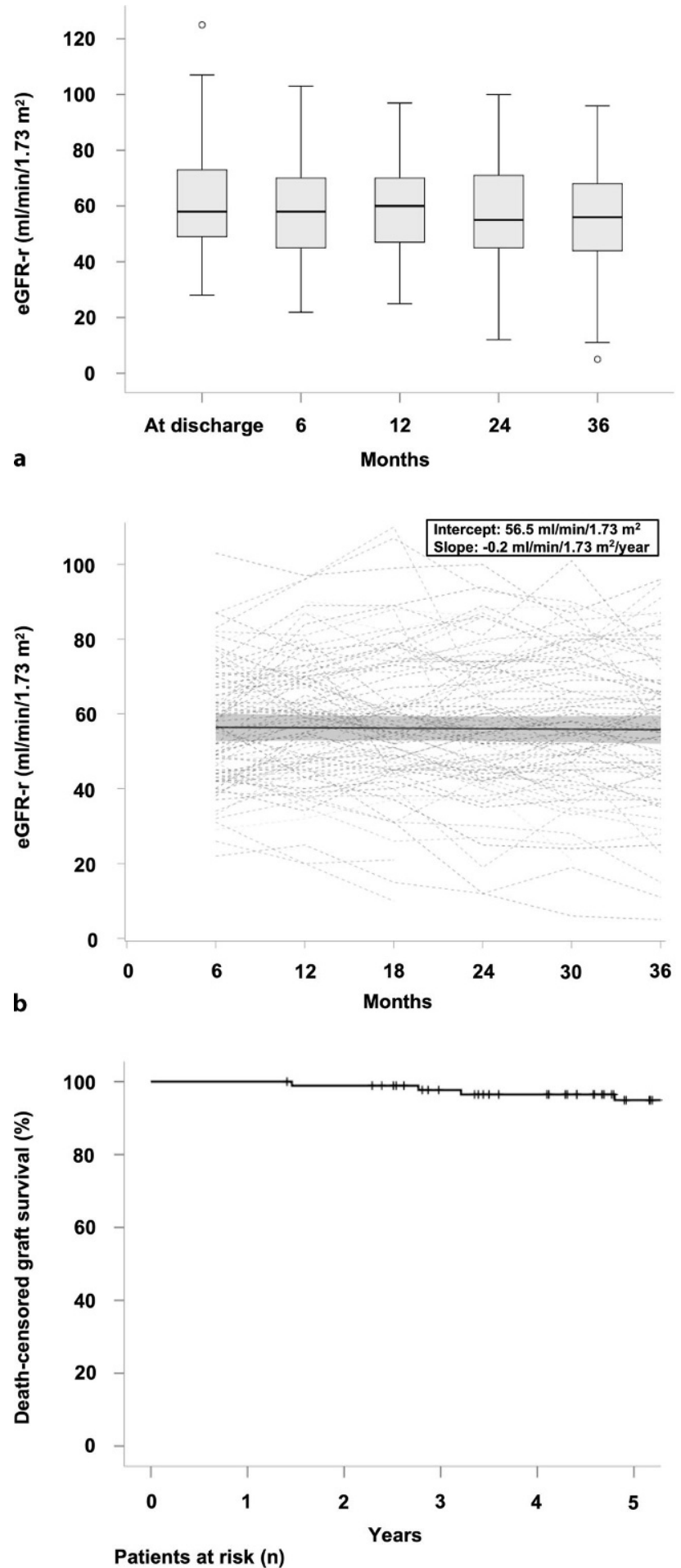

$\begin{array}{lllllll}C & 91 & 91 & 89 & 80 & 74 & 50\end{array}$

Fig. 2 Transplant and patient outcomes. a Boxplots indicate the median, interquartile range, minimum and maximum of recipient estimated glomerular filtration rate (eGFR-r) at hospital discharge and at $6,12,18,24$ and 36 months after transplantation. b Individual courses of eGFR-r (dashed lines) as well as its estimated mean (solid line) and the 95\% confidence interval (grey area) computed from an unadjusted mixed model are shown for a period between 6 months (intercept) and 3 years after transplantation. c Kaplan-Meier curves show death-censored graft survival over a period of 5 years 

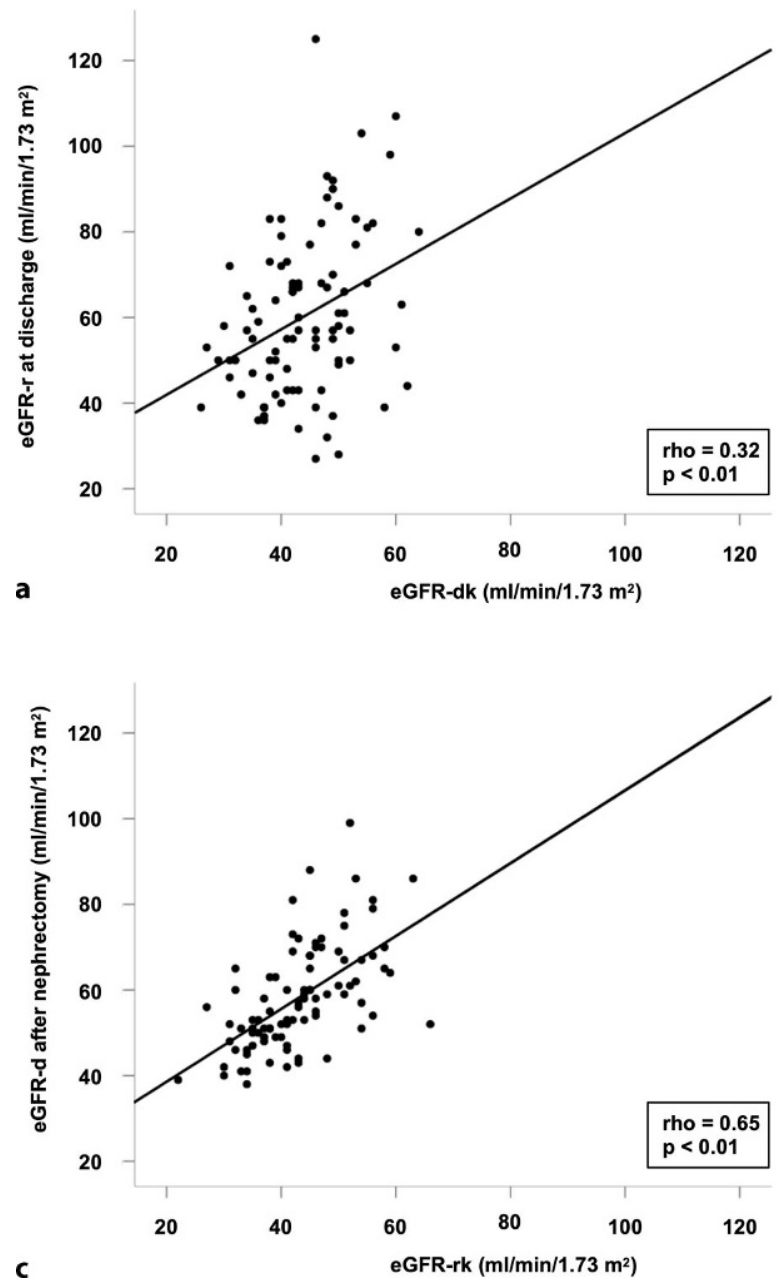

Fig. 3 Correlations between a predonation estimated glomerular filtration (eGFR) of the donated kidney (eGFR-dk) and recipient eGFR (eGFR-r) at discharge, b predonation total donor eGFR (eGFR-dt) and eGFR-r at discharge, c eGFR of the remaining donor kidney (eGFR-rk) and donor eGFR (eGFR-d) 1 week after nephrectomy, as well as d predonation eGFR-dt

$(P=0.14)$ or its slope $(P=0.52)$. In this model, however, increasing $\mathrm{LD}$ age showed a marginal association with lower baseline eGFR-r $(-0.5$ ( -1 to 0$) \mathrm{mL} / \mathrm{min} / 1.73 \mathrm{~m}^{2}$ mean estimated decrease per year; $P=0.05$ ). As in the first model, ABMR occurrence had a strong impact on eGFR-r slope (mean estimated annual decline: $-5.7(-10.4$ to -1.0$\left.) \mathrm{mL} / \mathrm{min} / 1.73 \mathrm{~m}^{2} ; P=0.02\right)$. In this model, only a slight effect was observed for BMI $(P=0.05)$.

\section{Discussion}

The primary objective of this study was to dissect the relative impact of LD kidney function on recipient baseline eGFR (intercept) at 6 months and eGFR slope. Major results of multivariable analysis were that the eGFR of donated kidneys, and in addition LD age, had an independent effect on allograft function at baseline, while there was no meaningful effect on eGFR slope. In line with earlier studies [23-25], ABMR
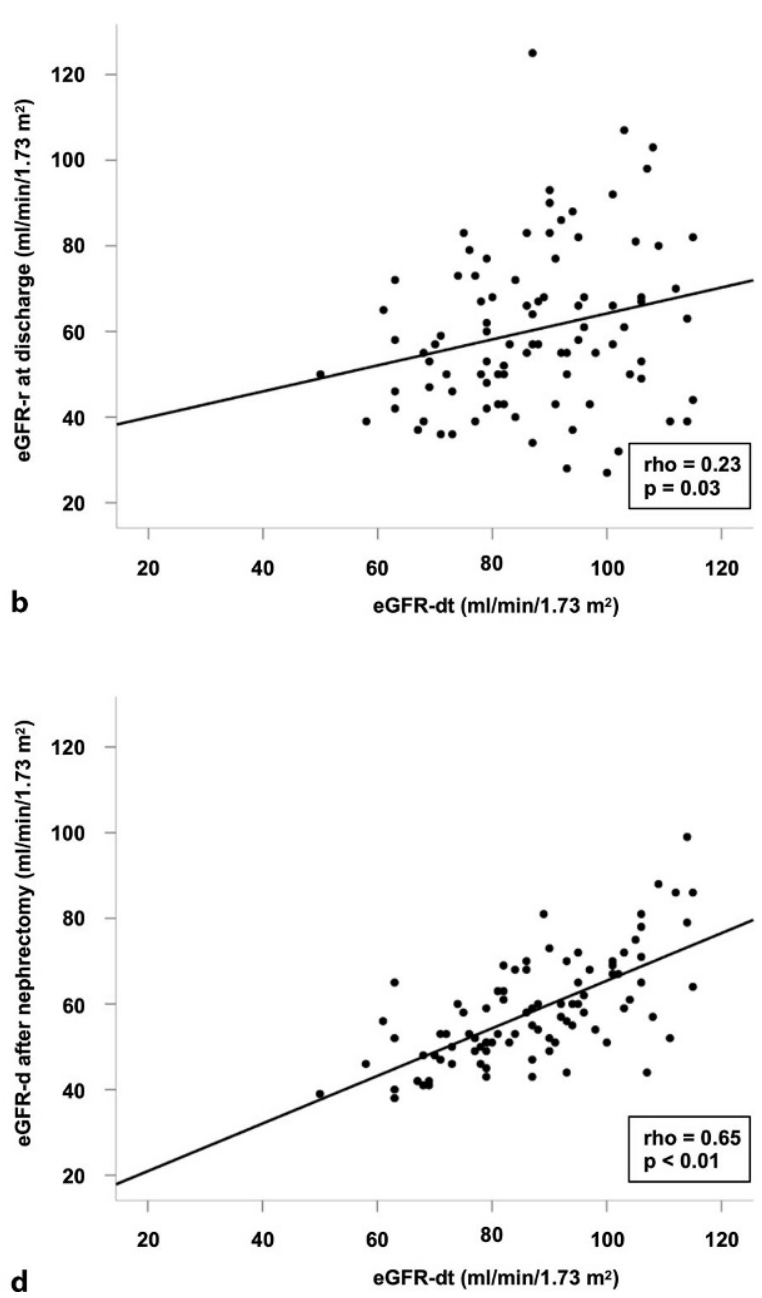

and eGFR-d 1 week after nephrectomy. Data are visualized by scatter plots and corresponding regression lines (solid lines) demonstrating the correlations between donor and recipient eGFR values. For statistical evaluation, test results were compared using Spearman's rank correlation analysis

was the dominant cause of transplant functional decline, with an associated mean eGFR slope of approximately $-6 \mathrm{~mL} / \mathrm{min} / 1.73 \mathrm{~m}^{2}$ per year as compared to $-0.2 \mathrm{~mL} / \mathrm{min} / 1.73 \mathrm{~m}^{2}$ per year in the overall cohort.

LD kidney transplantation is the best available treatment option for patients with ESRD, allowing for excellent clinical outcomes, with 1- and 5-year graft survival rates of $96 \%$ and $87 \%$, respectively shown for Europe [26]. Due to substantial demographical changes, however, the demand for donor organs is constantly rising. In recent years, there has been a progressive increase in the use of older $\mathrm{LD}$, who often present with additional risk factors, such as obesity, hypertension or subnormal GFR levels (even below $60 \mathrm{~mL} / \mathrm{min} / 1.73 \mathrm{~m}^{2}$ ) [4]. A trend towards the use of marginal donors raises major safety concerns regarding long-term LD outcomes. In addition, such variables, most prominently $\mathrm{LD}$ age and renal function, may also be important independent correlates of recipient allograft function. Previous studies have 
Table 4 Mixed linear model to evaluate the impact of the function of the donated kidney on recipient eGFR

\begin{tabular}{|c|c|c|c|c|}
\hline \multirow[t]{2}{*}{ Parameters } & \multicolumn{2}{|l|}{ Reduced model ${ }^{\mathrm{a}}$} & \multicolumn{2}{|l|}{ Multivariable model $^{b}$} \\
\hline & Estimate $(95 \% \mathrm{Cl})$ & $P$ value & Estimate $(95 \% \mathrm{Cl})$ & $P$ value \\
\hline \multicolumn{5}{|l|}{ Impact on recipient baseline eGFR, $\mathrm{mL} / \mathrm{min} / 1.73 \mathrm{~m}^{2}$} \\
\hline eGFR-dk ${ }^{\mathrm{c}}$, per mL/min/1.73 $\mathrm{m}^{2}$ & \multicolumn{2}{|c|}{ Changes with each calculation } & $0.6(0.1$ to 1.1$)$ & 0.02 \\
\hline \multicolumn{5}{|l|}{ Recipient variables } \\
\hline Age, per year & $-0.2(-0.5$ to 0$)$ & 0.06 & $-0.2(-0.4$ to 0.1$)$ & 0.23 \\
\hline Female sex, yes vs. no & $3.5(-4.7$ to 11.7$)$ & 0.40 & - & - \\
\hline Preemptive transplantation, yes vs. no & $1.9(-6.4$ to 10.3$)$ & 0.65 & - & - \\
\hline Recipient of a re-transplant, yes vs. no & $4.7(-8$ to 17.3$)$ & 0.46 & - & - \\
\hline ABO/HLA incompatible transplantation, yes vs. no & $1.3(-11.2$ to 13.9$)$ & 0.83 & - & - \\
\hline Sum of HLA mismatch in A, B and DR, per $n$ & $-0.9(-3.5$ to 1.8$)$ & 0.52 & - & - \\
\hline IL-2R antibody induction, yes vs. no & $-7.5(-20.5$ to 5.5$)$ & 0.26 & - & - \\
\hline Tacrolimus-based immunosuppression, yes vs. no & $2.6(-7.5$ to 12.6$)$ & 0.61 & - & - \\
\hline Belatacept-based immunosuppression, yes vs. no & $-0.3(-15.8$ to 15.3$)$ & 0.97 & - & - \\
\hline \multicolumn{5}{|l|}{ LD variables } \\
\hline Age, per year & $-0.5(-0.9$ to 0$)$ & 0.04 & $-0.4(-0.9$ to 0$)$ & 0.08 \\
\hline Female sex, yes vs. no & $-6.4(-14.5$ to 1.8$)$ & 0.12 & $-3.8(-12.0$ to 4.3$)$ & 0.35 \\
\hline BMI, per kg/m² & $0.8(-0.2$ to 1.8$)$ & 0.133 & $0.6(-0.4-1.6)$ & 0.24 \\
\hline Living-related, yes vs. no & $-2.5(-10.3$ to 5.4$)$ & 0.53 & - & - \\
\hline Abnormal MTT of donated kidneyd ${ }^{d}$, yes vs. no & $-6.8(-14.4$ to 0.9$)$ & 0.08 & $-6.2(-13.5$ to 1.2$)$ & 0.10 \\
\hline \multicolumn{5}{|l|}{ Biopsy-proven rejection } \\
\hline TCMR, yes vs. no & $0.1(-9.5$ to 9.7$)$ & 0.99 & $-2.0(-11.3$ to 7.2$)$ & 0.66 \\
\hline ABMR, yes vs. no & $0.5(-11.9$ to 12.8$)$ & 0.94 & $1.6(-10.1$ to 133$)$ & 0.79 \\
\hline \multicolumn{5}{|l|}{ Impact on recipient eGFR slope, $\mathrm{mL} / \mathrm{min} / 1.73 \mathrm{~m}^{2} /$ year } \\
\hline eGFR-dk ${ }^{\mathrm{c}}$, per $\mathrm{mL} / \mathrm{min} / 1.73 \mathrm{~m}^{2}$ & \multicolumn{2}{|c|}{ Changes with each calculation } & $-0.1(-0.3$ to 0.1$)$ & 0.27 \\
\hline \multicolumn{5}{|l|}{ Recipient variables } \\
\hline Age, per year & 0.1 (0 to 0.2$)$ & 0.26 & $0(-0.1$ to 0.1$)$ & 0.39 \\
\hline Female sex, yes vs. no & $-2.1(-5.2$ to 1.1$)$ & 0.20 & - & - \\
\hline Preemptive transplantation, yes vs. no & $1.1(-2.1$ to 4.4$)$ & 0.49 & - & - \\
\hline Recipient of a re-transplant, yes vs. no & $0.9(-4.2$ to 6$)$ & 0.73 & - & - \\
\hline ABO/HLA incompatible transplantation, yes vs. no & $-0.5(-5.4$ to 4.4$)$ & 0.85 & - & - \\
\hline Sum of HLA mismatch in A, B and DR, per $n$ & $0.3(-0.8$ to 1.3$)$ & 0.59 & - & - \\
\hline IL-2R antibody induction, yes vs. no & $1.4(-3.5$ to 6.3$)$ & 0.58 & - & - \\
\hline Tacrolimus-based immunosuppression, yes vs. no & $2.5(-1.4$ to 6.4$)$ & 0.20 & - & - \\
\hline Belatacept-based immunosuppression, yes vs. no & $-0.9(-6.7$ to 4.9$)$ & 0.77 & - & - \\
\hline \multicolumn{5}{|l|}{ LD variables } \\
\hline Age, per year & $-0.2(-0.3$ to 0$)$ & 0.09 & $-0.2(-0.4$ to 0$)$ & 0.07 \\
\hline Female sex, yes vs. no & $2.5(-0.6$ to 5.7$)$ & 0.11 & $0.9(-2.4$ to 4.1$)$ & 0.60 \\
\hline BMI, per kg/m² & $-0.4(-0.8$ to -0.01$)$ & 0.03 & $-0.5(-0.8$ to -0$)$ & 0.04 \\
\hline Living-related, yes vs. no & $-0.2(-3.3$ to 2.8$)$ & 0.87 & - & - \\
\hline Abnormal MTT of donated kidney ${ }^{d}$, yes vs. no & $0.6(-2.4$ to 3.6$)$ & 0.71 & $0.9(-2.1$ to 3.8$)$ & 0.56 \\
\hline \multicolumn{5}{|l|}{ Biopsy-proven rejection } \\
\hline TCMR, yes vs. no & $-2.8(-6.5$ to 0.9$)$ & 0.13 & $-1.2(-4.9$ to 2.5$)$ & 0.51 \\
\hline ABMR, yes vs. no & $-5.3(-10$ to -0.6$)$ & 0.03 & $-5.8(-10.4$ to -1.2$)$ & 0.01 \\
\hline \multicolumn{5}{|c|}{$\begin{array}{l}\text { ABMR antibody-mediated rejection, } B M I \text { body mass index, } C I \text { confidence interval, eGFR estimated glomerular filtration rate, eGFR-dk eGFR of the donated } \\
\text { kidney, IL-2R interleukin-2 receptor, } L D \text { live donor, } M T T \text { mean transit time, TCMRT cell-mediated rejection } \\
\text { aIn the reduced model recipient eGFR values from } 6 \text { to } 36 \text { months were used as dependent variable. eGFR-dk and time were included in each calculation. Other } \\
\text { variables were added one by one. Slope estimates additionally considered interactions of variables with time } \\
\text { bThe multivariable model included eGFR-dk and time. Additionally, the model was expanded by variables with a } P \text { value of }<0.157 \text { for their impact on recipient } \\
\text { baseline eGFR or its slope in the reduced model } \\
{ }^{c} \text { eGFR-dk was calculated from total donor eGFR on the basis of the relative kidney function determined in isotope nephrography } \\
\text { dMTT values of } 1.9 \text { to } 2.9 \text { min were considered normal }\end{array}$} \\
\hline
\end{tabular}




\section{original article}

Table 5 Mixed linear model to evaluate the impact of total LD kidney function on recipient eGFR

\begin{tabular}{|c|c|c|c|c|}
\hline \multirow[t]{2}{*}{ Parameters } & \multicolumn{2}{|l|}{ Reduced model ${ }^{\mathrm{a}}$} & \multicolumn{2}{|l|}{ Multivariable model $^{b}$} \\
\hline & Estimate $(95 \% \mathrm{Cl})$ & $P$ value & Estimate $(95 \% \mathrm{Cl})$ & $P$ value \\
\hline \multicolumn{5}{|l|}{ Impact on recipient baseline eGFR, $\mathrm{mL} / \mathrm{min} / 1.73 \mathrm{~m}^{2}$} \\
\hline eGFR-dt, per $\mathrm{mL} / \mathrm{min} / 1.73 \mathrm{~m}^{2}$ & \multicolumn{2}{|c|}{ Changes with each calculation } & $0.2(-0.1$ to 0.5$)$ & 0.14 \\
\hline \multicolumn{5}{|l|}{ Recipient variables } \\
\hline Age, per year & $-0.3(-0.5$ to 0$)$ & 0.04 & $-0.2(-0.4$ to 0.1$)$ & 0.20 \\
\hline Female sex, yes vs. no & $4.8(-3.4$ to 13.1$)$ & 0.25 & - & - \\
\hline Preemptive transplantation, yes vs. no & $1.9(-6.5$ to 10.4$)$ & 0.65 & - & - \\
\hline Recipient of a re-transplant, yes vs. no & $6.4(-6.4$ to 19.1$)$ & 0.32 & - & - \\
\hline ABO/HLA incompatible transplantation, yes vs. no & $0.2(-12.5$ to 13$)$ & 0.97 & - & - \\
\hline Sum of HLA mismatch in A, B and DR, per $n$ & $-0.7(-3.4$ to 2$)$ & 0.60 & - & - \\
\hline IL-2R antibody induction, yes vs. no & $-7(-20.2$ to 6.2$)$ & 0.29 & - & - \\
\hline Tacrolimus-based immunosuppression, yes vs. no & $1.3(-8.9$ to 11.5$)$ & 0.80 & - & - \\
\hline Belatacept-based immunosuppression, yes vs. no & $-0.1(-15.8$ to 15.7$)$ & 0.99 & - & - \\
\hline \multicolumn{5}{|l|}{ LD variables } \\
\hline Age, per year & $-0.6(-1.1$ to -0.1$)$ & 0.02 & $-0.5(-1$ to 0$)$ & 0.05 \\
\hline Female sex, yes vs. no & $-7.1(-15.3$ to 1.1$)$ & 0.09 & $-4.6(-12.8$ to 3.7$)$ & 0.28 \\
\hline BMI, per kg/m² & $0.72(-0.3$ to 1.7$)$ & 0.16 & $0.5(-0.5$ to 1.5$)$ & 0.30 \\
\hline Living-related, yes vs. no & $-3.2(-11.2$ to 4.7$)$ & 0.42 & - & - \\
\hline Abnormal MTT of donated kidneyc ${ }^{\mathrm{C}}$, yes vs. no & $-8.3(-16$ to -0.5$)$ & 0.04 & $-6.6(-14.2$ to 0.9$)$ & 0.08 \\
\hline \multicolumn{5}{|l|}{ Biopsy-proven rejection } \\
\hline TCMR, yes vs. no & $1.4(-8.4$ to 11.3$)$ & 0.77 & $-1.3(-10.7$ to 8.1$)$ & 0.78 \\
\hline ABMR, yes vs. no & $0.8(-11.9$ to 13.5$)$ & 0.90 & $1.4(-10.6$ to 13.4$)$ & 0.81 \\
\hline \multicolumn{5}{|l|}{ Impact on recipient eGFR slope, $\mathrm{mL} / \mathrm{min} / 1.73 \mathrm{~m}^{2} /$ year } \\
\hline eGFR-dt, per mL/min $/ 1.73 \mathrm{~m}^{2}$ & \multicolumn{2}{|c|}{ Changes with each calculation } & $0(-0.1$ to 0.1$)$ & 0.52 \\
\hline \multicolumn{5}{|l|}{ Recipient variables } \\
\hline Age, per year & $0.1(0$ to 0.2$)$ & 0.21 & $0(-0.1$ to 0.1$)$ & 0.20 \\
\hline Female sex, yes vs. no & $-2.2(-5.3$ to 1$)$ & 0.17 & - & - \\
\hline Preemptive transplantation, yes vs. no & $1.3(-2$ to 4.5$)$ & 0.44 & - & - \\
\hline Recipient of a re-transplant, yes vs. no & $0.5(-4.6$ to 5.6$)$ & 0.84 & - & - \\
\hline ABO/HLA incompatible transplantation, yes vs. no & $-0.5(-5.5$ to 4.4$)$ & 0.84 & - & - \\
\hline Sum of HLA mismatch in A, B and DR, per $n$ & $0.3(-0.8$ to 1.3$)$ & 0.60 & - & - \\
\hline IL-2R antibody induction, yes vs. no & $1.1(-3.8$ to 6.1$)$ & 0.65 & - & - \\
\hline Tacrolimus-based immunosuppression, yes vs. no & $2.6(-1.3$ to 6.5$)$ & 0.19 & - & - \\
\hline Belatacept-based immunosuppression, yes vs. no & $-1(-6.8$ to 4.8$)$ & 0.74 & - & - \\
\hline \multicolumn{5}{|l|}{ LD variables } \\
\hline Age, per year & $-0.1(-0.3$ to 0.1$)$ & 0.18 & $-0.2(-0.4$ to 0$)$ & 0.11 \\
\hline Female sex, yes vs. no & $2.7(-0.4$ to 5.9$)$ & 0.09 & $1.0(-2.2$ to 4.3$)$ & 0.53 \\
\hline BMI, per kg/m² & $-0.4(-0.8$ to 0$)$ & 0.03 & $-0.4(-0.8$ to 0$)$ & 0.05 \\
\hline Living-related, yes vs. no & $-0.3(-3.3$ to 2.8$)$ & 0.87 & - & - \\
\hline Abnormal MTT of donated kidney ${ }^{\mathrm{C}}$, yes vs. no & $0.6(-2.4$ to 3.6$)$ & 0.70 & $1.0(-2.0$ to 4.0$)$ & 0.53 \\
\hline \multicolumn{5}{|l|}{ Biopsy-proven rejection } \\
\hline TCMR, yes vs. no & $-2.7(-6.5$ to 1$)$ & 0.15 & $-1.4(-5.1$ to 2.4$)$ & 0.47 \\
\hline ABMR, yes vs. no & $-5.1(-9.8$ to -0.3$)$ & 0.04 & $-5.7(-10.4$ to -1.0$)$ & 0.02 \\
\hline
\end{tabular}

$A B M R$ antibody-mediated rejection, BMI body mass index, $C l$ confidence interval, eGFR estimated glomerular filtration rate, eGFR-dt total donor eGFR, IL-2R interleukin-2 receptor, LD live donor, MTT mean transit time, TCMRT cell-mediated rejection

aIn the reduced model recipient eGFR values from 6 to 36 months were used as dependent variable. eGFR-dt and time were included in each calculation. Other variables were added one by one. Slope estimates additionally considered interactions of variables with time

${ }^{\mathrm{b}}$ The multivariable model included eGFR-dt and time. Additionally, the model was expanded by variables with a $P$ value of $<0.157$ for their impact on recipient baseline eGFR or its slope in the reduced model

${ }^{\mathrm{C} M T T}$ values of 1.9-2.9 min were considered normal 
shown that recipients of kidneys from older donors are at an increased risk of delayed graft function, graft failure and death [5, 6]. Similar associations have been observed for subnormal predonation GFR, but in smaller recipient cohorts and in less well-designed studies [13].

For our study, we have chosen baseline eGFR-r at 6 months and eGFR-r slope calculated from 6 -monthly measurements as dependent variables in mixed linear models. There is increasing evidence that the extent of eGFR decline over time may serve as a valuable surrogate endpoint for long-term renal survival, in both transplantation [14, 15] and native kidney disease $[27,28]$. For example, evaluating a consecutive cohort of 508 non-sensitized DD or LD renal allograft recipients, Wiebe et al. [23] described a close interrelationship between eGFR and long-term graft survival. Focusing on a specific subgroup of renal allograft recipients who developed de novo donorspecific antibodies ( $d n \mathrm{DSA})$, a highly significant $6 \%$ increase in the risk of post- $d n \mathrm{DSA}$ graft loss was calculated for each $1 \mathrm{~mL} / \mathrm{min} / 1.73 \mathrm{~m}^{2}$ decease in eGFR at 3 years postsubclinical $d n$ DSA onset [23].

We identified donor kidney function and, in accordance with previous studies $[9,10]$, donor age as independent predictors of baseline eGFR-r, reinforcing the usefulness of these parameters for risk stratification of organs from potential kidney donors. In our cohort of LD kidney transplants, eGFR-dk was associated with a mean estimated increase in recipient baseline eGFR of $0.6 \mathrm{~mL} / \mathrm{min} / 1.73 \mathrm{~m}^{2}$ per unit and increasing donor age was associated with a marginal decrease in recipient baseline eGFR. In contrast, we found no significant effect of total kidney function in the eGFRdt-adjusted model. This result indicates a diagnostic benefit of ING for assessment of functional side distribution in the context of LD evaluation; however, we are aware of the limited sample size which may have precluded detection of subtle differences. For another ING-based parameter-MTT to quantify the dynamics of parenchymal tracer transit-we found no association with any of the endpoints, suggesting that this parameter may have a limited diagnostic value in the evaluation of normal functioning kidneys; however, impaired renal transit may help to dissect certain disease states, such as acute tubular injury or cyclosporine toxicity in renal transplants [29].

Interestingly, while there was a marginal effect of BMI, our study did not reveal a significant effect of LD kidney function (and age) on the slope of eGFR-r. This finding was unexpected considering the potential functional impact of a limited renal functional reserve associated with lower donor GFR, which may ultimately cause injury due to hyperfiltration in remaining nephrons [30]; however, we want to point out that our local standard did not accept donors with an adjusted measured GFR (or a urinary creatinine clearance) $<80 \mathrm{~mL} / \mathrm{min}$ and/or an unequal distribution of kidney function detected by ING $(>20 \%$ side differ- ence), and this policy resulted in the overall inclusion of donated kidneys with favorable baseline function (median eGFR-dk: 43 (IQR: 38-50) $\mathrm{mL} / \mathrm{min} / 1.73 \mathrm{~m}^{2}$; median relative function: 51 (48-54) \%). Our results are in line with an earlier analysis of 4488 patients, mostly DD recipients, where donor age had a significant impact on recipient eGFR at 12 months, but no influence on eGFR slope [9]. Perhaps as a result of inherent differences in case selection, which may also include marked differences in donor characteristics, other studies have revealed controversial results. For example, in a study by Issa et al. [8] changes in eGFR of LD kidney recipients over a period of 2 years after transplantation were estimated at $-8.76 \mathrm{~mL} / \mathrm{min} / 1.73 \mathrm{~m}^{2}$, if donors were aged $\geq 45$ years and at $-7.40 \mathrm{~mL} / \mathrm{min} / 1.73 \mathrm{~m}^{2}$, if donors had an unadjusted predonation eGFR of $<110 \mathrm{~mL} / \mathrm{min}$. Moreover, also in two other larger studies [7, 10], donor age was reported to be a significant determinant of progressive functional deterioration of renal allografts, in one of these studies [10], however, only beyond the first post-transplantation year.

A major finding of our study was that ABMR (10 recipients in our cohort) turned out to be the strongest predictor of annual eGFR-r decline. The diagnosis of acute or chronic active ABMR, the leading cause of graft failure in our cohort (six of nine recorded allograft losses), was found to be associated with a mean eGFR-r slope of approximately $-6 \mathrm{~mL} / \mathrm{min} / 1.73 \mathrm{~m}^{2}$ per year. This observation is consistent with the previous literature reinforcing a deleterious impact of ABMR on kidney allograft outcomes [31]. Few studies have analyzed the finding of $d n \mathrm{DSA}$ or diagnosis of ABMR in relation to the dynamics of eGFR decline. For example, Wiebe et al. [23] found an eGFR decline of -3.15 and $-5.61 \mathrm{~mL} / \mathrm{min} / 1.73 \mathrm{~m}^{2}$ per year in patients with subclinical $(n=19)$ and clinical $(n=45) d n \mathrm{DSA}$, respectively. Moreover, in a recent randomized controlled trial evaluating bortezomib in 44 subjects with late ABMR, eGFR slopes were about $-5 \mathrm{~mL} / \mathrm{min} / 1.73 \mathrm{~m}^{2}$ per year in both placebo and treatment groups [24]. Similar results (eGFR slope of approximately $-7 \mathrm{~mL} / \mathrm{min} / 1.73 \mathrm{~m}^{2}$ per year among 25 randomized subjects) were reported in a trial evaluating the effect of combined IVIG and rituximab in ABMR with transplant glomerulopathy [25]. The unfavorable course of allograft function in patients with ABMR, as opposed to TCMR, may reflect the current unavailability of effective therapeutic measures to counteract this type of rejection, in particular late ABMR associated with chronic irreversible injury [24, 25]. Our data reinforce the need for the establishment of effective measures to prevent or treat ABMR.

Our study has several inherent limitations. One major limitation is the comparatively small sample size, which was due to the monocentric study design and limited availability of ING data in our cohort. While we were able to dissect strong independent predictors of graft function evolution, our study may have 
not been sufficiently powered to detect subtle effects of some other potentially confounding variables, such as baseline immunosuppression (e.g. calcineurin inhibitors versus belatacept, which may delay progressive functional deterioration [32]). Another limitation is the intermediate-term follow-up (median 7 years), which in our cohort of LD kidney transplant recipients coincided with a low rate of graft loss (10\%). Therefore, based on previous studies, we have selected eGFR slope as a surrogate endpoint, which allowed us to detect relevant outcome differences even in (i) a smaller cohort and (ii) after a shorter followup period. Finally, it may also be considered a limitation that our analysis was based on serum creatininebased estimations of donor kidney function. Measured GFR was available only for half of the included LD and the resulting sample size would have been too small to detect meaningful effects. For our study, we have chosen the CKD-EPI equation, which, in contrast to other equations, such as the MDRD equation, may more accurately reflect the GFR in subjects with normal renal function [16].

While our results support that LD kidney function and age independently predict allograft function at baseline, we were not able to demonstrate a significant effect of these variables on the slope of recipient eGFR. In contrast, occurrence of ABMR turned out to be the strongest risk factor for accelerated loss of allograft function after LD kidney transplantation.

Acknowledgements The authors wish to thank Elisabeth Lehner for her valuable support in data collection and Peter Schaffarich for importing archived scintigraphicimaging data.

Funding Open access funding provided by Medical University of Vienna.

Conflict of interest M. Hamböck, A. Staudenherz, A. Kainz, B. Geist, M. Hecking, K. Doberer, M. Hacker, and G.A. Böhmig declare that they have no competing interests.

Open Access This article is licensed under a Creative Commons Attribution 4.0 International License, which permits use, sharing, adaptation, distribution and reproduction in any medium or format, as long as you give appropriate credit to the original author(s) and the source, provide a link to the Creative Commons licence, and indicate if changes were made. The images or other third party material in this article are included in the article's Creative Commons licence, unless indicated otherwise in a credit line to the material. If material is not included in the article's Creative Commons licence and your intended use is not permitted by statutory regulation or exceeds the permitted use, you will need to obtain permission directly from the copyright holder. To view a copy of this licence, visit http://creativecommons.org/licenses/by/4.0/.

\section{References}

1. Lentine KL, Kasiske BL, Levey AS, etal. KDIGO clinical practice guideline on the evaluation and care of living kidney donors. Transplantation. 2017;101(8SSuppl 1):S1-S109.

2. Andrews PA, Burnapp L, Manas D, et al. Summary of the British transplantation society/renal association U.K. guidelines for living donor kidney transplantation. Transplantation. 2012;93(7):666-73.

3. Grams ME, Sang Y, Levey AS, et al. Kidney-failure risk projection for the living kidney-donor candidate. N Engl J Med. 2016;374(5):411-21.

4. Reese PP, Feldman HI, McBride MA, Anderson K, Asch DA, Bloom RD. Substantial variation in the acceptance of medically complex live kidney donors across US renal transplant centers. Am J Transplant. 2008;8(10):2062-70.

5. Massie AB, Leanza J, Fahmy LM, et al. A risk index for living donor kidney transplantation. Am J Transplant. 2016;16(7):2077-84.

6. Sapir-Pichhadze R, Young A, Joseph Kim S. Living donor age and kidney transplant outcomes: an assessment of risk across the age continuum. Transpl Int. 2013;26(5):493-501.

7. Gill JS, Tonelli M, Mix CH, Pereira BJ. The change in allograft function among long-term kidney transplant recipients. JAm Soc Nephrol. 2003;14(6):1636-42.

8. Issa N, Stephany B, Fatica R, et al. Donor factors influencing graft outcomes in live donor kidney transplantation. Transplantation. 2007;83(5):593-9.

9. Marcen R, Morales JM, Fernandez-Rodriguez A, et al. Longterm graft function changes in kidney transplant recipients. NDT Plus. 2010;3(2):ii2-ii8.

10. Noppakun K, Cosio FG, Dean PG, Taler SJ, Wauters R, Grande JP. Living donor age and kidney transplant outcomes. Am J Transplant. 2011;11(6):1279-86.

11. SmitsJM, PersijnGG, vanHouwelingenHC, ClaasFH, FreiU. Evaluation of the eurotransplant senior program. The results of the first year. Am J Transplant. 2002;2(7):664-70.

12. Norden G, LennerlingA, Nyberg G. Low absoluteglomerular filtration rate in theliving kidney donor: a riskfactor for graft loss. Transplantation. 2000;70(9):1360-2.

13. Iordanous Y, Seymour N, Young A, et al. Recipient outcomes for expanded criteria living kidney donors: the disconnect between current evidence and practice. Am J Transplant. 2009;9(7):1558-73.

14. Clayton PA, Lim WH, Wong G, Chadban SJ. Relationship between eGFR decline and hard outcomes after kidney transplants. JAm Soc Nephrol. 2016;27(11):3440-6.

15. Park WD, Larson TS, Griffin MD, Stegall MD. Identification and characterization of kidney transplants with good glomerular filtration rate at 1 year but subsequent progressive loss of renal function. Transplantation. 2012;94(9):931-9.

16. Levey AS, Stevens LA, Schmid CH, et al. A new equation to estimate glomerular filtration rate. Ann Intern Med. 2009;150(9):604-12.

17. Geist BK, Diemling M, Staudenherz A. Glomerular filtration rate and error calculation based on the slopeintercept method with chromium-51 ethylenediaminetetraacetic acid via a new clinical software: GFRcalc. Med Princ Pract. 2016;25(4):368-73.

18. Taylor AT, Brandon DC, de Palma D, et al. SNMMI procedure standard/EANM practice guideline for diuretic renal scintigraphy in adults with suspected upper urinary tract obstruction 1.0. Semin Nucl Med. 2018;48(4):377-90.

19. Geist BK, Dobrozemsky G, Samal M, Schaffarich MP, Sinzinger H, Staudenherz A. WWSSF-a worldwide study on radioisotopic renal split function: reproducibility of renal split function assessment in children. Nucl Med Commun. 2015;36(12):1233-8.

20. Durand E, Blaufox MD, Britton KE, et al. International scientific committee of radionuclides in nephrourology (ISCORN) consensus on renal transit time measurements. Semin Nucl Med. 2008;38(1):82-102. 
21. Loupy A, Haas M, Solez K, et al. The Banff 2015 kidney meeting report: current challenges in rejection classification and prospects for adopting molecular pathology. Am J Transplant. 2017;17(1):28-41.

22. Teräsvirta T, Mellin I. Model selection criteria and model selection tests in regression models. Scand Stat Theory Appl. 1986;13:159-71.

23. Wiebe C, Gibson IW, Blydt-Hansen TD, et al. Rates and determinants of progression to graft failure in kidney allograft recipients with de novo donor-specific antibody. Am J Transplant. 2015;15(11):2921-30.

24. Eskandary F, Regele H, Baumann L, et al. Arandomized trial of bortezomib in late antibody-mediated kidney transplant rejection. JAm Soc Nephrol. 2018;29(2):591-605.

25. Moreso F, Crespo M, RuizJC, etal. Treatment of chronic antibody mediated rejection with intravenous immunoglobulins and rituximab: a multicenter, prospective, randomized, double-blind clinical trial. Am J Transplant. 2018;18(4):927-35.

26. Wang JH, Skeans MA, Israni AK. Current status of kidney transplant outcomes: dying to survive. Adv Chronic Kidney Dis. 2016;23(5):281-6.
27. Coresh J, Turin TC, Matsushita K, et al. Decline in estimated glomerular filtration rate and subsequent risk of end-stage renal disease and mortality. JAMA. 2014;311(24):2518-31.

28. Thompson A, Lawrence J, Stockbridge N. GFR decline as an end point in trials of CKD: a viewpoint from the FDA. Am J Kidney Dis. 2014;64(6):836-7.

29. SanchesA,EtchebehereEC, MazzaliM, etal. The accuracy of $(99 \mathrm{~m}) \mathrm{Tc}$-DTPA scintigraphy in the evaluation of acute renal graft complications. Int Braz J Urol. 2003;29(6):507-16.

30. Hostetter TH, Olson JL, Rennke HG, Venkatachalam MA, Brenner BM. Hyperfiltration in remnant nephrons: a potentially adverse response to renal ablation. Am J Physiol. 1981;241(1):F85-93.

31. Loupy A, Lefaucheur C. Antibody-mediated rejection of solid-organ allografts. N Engl J Med. 2018;379(12):1150-60.

32. Vincenti F, Rostaing L, Grinyo J, et al. Belatacept and longterm outcomes in kidney transplantation. N Engl J Med. 2016;374(4):333-43.

Publisher's Note Springer Nature remains neutral with regard to jurisdictional claims in published maps and institutional affiliations. 